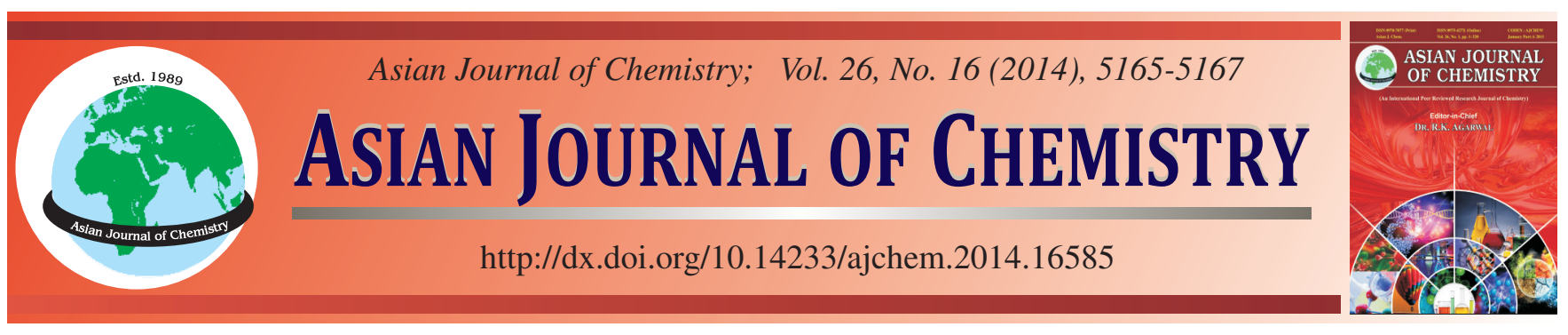

\title{
Synthesis and Antimicrobial Activities of 2,5-Substituent Hydroquinone Derivatives
}

\author{
Hui-Lian LiU* and Qing-ZhONG WANG
}

College of Biological and Agricultural Engineering, Weifang University, Key Laboratory of Biochemistry and Molecular Biology in Universities of Shandong, Weifang 261061, Shandong Province, P.R. China

*Corresponding author: Tel/Fax: +86 536 8785288; E-mail: huilian.liu@163.com

Received: 26 October 2013;

Accepted: 18 March 2014;

Published online: 28 July 2014;

AJC-15653

\begin{abstract}
A series of 2,5-substituent hydroquinone derivatives were designed and synthesized. Their structures were identified by elemental analysis, ${ }^{1} \mathrm{H}$ NMR, IR spectra. Their assayed antibacterial (Escherichia coli, Bacillus subtilis) and antifungal (Candida albicans) activities were also evaluated by MTT (3-(4,5-dimethylthiazol-2-yl)-2,5-diphenyl tetrazolium bromide) method. The results of biological test showed compounds 1 and $\mathbf{1 0}$ have favorable antimicrobial activity with MICs of 20.5, 24.6, 18.9 and 28.6 $\mu \mathrm{g} / \mathrm{mL}$ against Escherichia coli and Bacillus subtilis, respectively.
\end{abstract}

Keywords: Hydroquinone derivatives, Antimicrobial activities, Structure-activity relationships.

\section{INTRODUCTION}

As an important industrial organic compound, 2,5-substituted hydroquinone was used in rubber manufacture ${ }^{1}$, plastic additives, dyes and specialty thermosetting resins ${ }^{2-4}$. They occurred in key biological processes as diverse as the oxidative maintenance of biological amine levels ${ }^{5}$, tissue (collagen and elastin) formation ${ }^{6}$ photosynthesis ${ }^{7}$ and aerobic (mitochondrial) respiration ${ }^{8} .2,5$-Substituted hydroquinone are vital for all life, occurring in key biological processes as diverse as the oxidative maintenance of biological amine levels. Their derivatives are the basic structure of the quinones, which is not only found in bacteria, plants and arthropods but are also the base for a large number of chemical derivatives with pharmacological applications ${ }^{9-11}$. However, the antibacterial activity of 2,5-substituted hydroquinone was less reported. In this paper, the compounds were prepared to study their antibacterial activity against Bacillus subtilis, Escherichia coli and Candida albicans. The results of this study may be useful to researchers attempting to gain more understanding of the antimicrobial activity of 2,5-substituted hydroquinone compounds. The synthesis route is described in Scheme-I.

\section{EXPERIMENTAL}

All chemicals and reagents were obtained from commercial sources and used without further purification. Melting points were measured on a Boetius micro melting point apparatus. The IR spectra were recorded in the $4000-400 \mathrm{~cm}^{-1}$ region using $\mathrm{KBr}$ pellets on a Nicolet $170 \mathrm{SX}$ spectrophotometer.<smiles>[X]C(=O)c1cc(O)c(C([X])=O)cc1O</smiles>

(1)

(2)

Scheme-I

All the NMR spectra were recorded on a Bruker DRX500 model spectrometer in $\mathrm{CDCl}_{3}$. Chemical shifts for ${ }^{1} \mathrm{H}$ NMR spectra were reported in parts per million to residual solvent protons.

General synthetic procedure: To a $100 \mathrm{~mL}$ flask $20 \mathrm{~mL}$ concentrated sulfuric acid, 0.05 mol 2,5-substituent 1,4cyclohexanedione was added dropwise with stirring. The solid of 2,5-substituent 1,4-cyclohexanedione suspend on the concentrated sulfuric acid at first, gradually dissolved later. The reaction was maintained until the mixture was turned to clear solution for 4-5 h. Then add above clear solution into ice-water with rapidly stirring. The precipitation was formed and filtered. The light yellow or white solid produces have been obtained by recrystallized from EtOH. The yields of ten synthesized compounds are listed in Table- 1 .

2,5-Dimethylformate hydroquinone (1): Yield: $98 \%$. m.p. $155-157{ }^{\circ} \mathrm{C} ;{ }^{1} \mathrm{H}$ NMR $\left(400 \mathrm{~Hz}, \mathrm{CDCl}_{3}\right): \delta 11.98(\mathrm{~s}, 2 \mathrm{H})$, $7.26(\mathrm{~s}, 2 \mathrm{H}), 3.13$ (s, 6H); IR (KBr, $\left.v_{\max }, \mathrm{cm}^{-1}\right): 3168,2961$, 2910, 1669, 1347, 1224, 1073, 812, 796. Elemental analysis 


\begin{tabular}{|c|c|c|c|}
\hline \multicolumn{4}{|c|}{$\begin{array}{c}\text { TABLE-1 } \\
\text { PRODUCTS AND YIELDS FROM THE DIFFERENT } \\
\text { STARTING MATERIALS IN } \mathrm{K}_{2} \mathrm{CO}_{3} / \text { EtOH SYSTEM }\end{array}$} \\
\hline Entry & X Groups & Melt point $\left({ }^{\circ} \mathrm{C}\right)$ & Yield (\%) \\
\hline 1 & $\mathrm{CH}_{3} \mathrm{O}$ & $155-157$ & 98.0 \\
\hline 2 & $\mathrm{CH}_{3} \mathrm{CH}_{2} \mathrm{O}$ & 136.4 & 98.2 \\
\hline 3 & $\mathrm{CH}_{3} \mathrm{CH}_{2} \mathrm{CH}_{2} \mathrm{O}$ & 100.4 & 98.3 \\
\hline 4 & $\left(\mathrm{CH}_{3}\right)_{2} \mathrm{CHO}$ & $136.2-137.1$ & 97.0 \\
\hline 5 & $\mathrm{CH}_{3}\left(\mathrm{CH}_{2}\right)_{2} \mathrm{CH}_{2} \mathrm{O}$ & 101.8 & 87.9 \\
\hline 6 & $\mathrm{CH}_{3} \mathrm{CH}_{2}\left(\mathrm{CH}_{3}\right) \mathrm{CHO}$ & $>200$ & 85.8 \\
\hline 7 & $\left(\mathrm{CH}_{3}\right)_{2} \mathrm{CHCH}_{2} \mathrm{O}$ & 111 & 86.7 \\
\hline 8 & $\mathrm{CH}_{3}\left(\mathrm{CH}_{2}\right)_{3} \mathrm{CH}_{2} \mathrm{O}$ & $104.7-105$ & 83.6 \\
\hline 9 & $\left(\mathrm{CH}_{3}\right)_{2} \mathrm{CHCH}_{2} \mathrm{CH}_{2} \mathrm{O}$ & $48-49$ & 80.1 \\
\hline 10 & —o & $207-208.2$ & 79.2 \\
\hline
\end{tabular}

[Anal. calcd. (\%) for $\mathrm{C}_{10} \mathrm{H}_{10} \mathrm{O}_{6}$ ]: C 53.10, $\mathrm{H} 4.46$; Found (\%): C 53.02, H 4.22.

2,5-Diethylformate hydroquinone (2): Yield: $98.2 \%$. m.p. $136.4{ }^{\circ} \mathrm{C} ;{ }^{1} \mathrm{H}$ NMR $\left(400 \mathrm{~Hz}, \mathrm{CDCl}_{3}\right): \delta 9.87(\mathrm{~s}, 2 \mathrm{H}), 7.27$ (s, 2H), 4.39-4.31 (m, 4H), 1.34-1.31 (t, 6H); IR (KBr, $v_{\max }$, $\mathrm{cm}^{-1}$ ): 3296, 2990, 2907, 1685, 1402, 1197, 1099, 802, 792. Elemental analysis [Anal. calcd. (\%) for $\mathrm{C}_{12} \mathrm{H}_{14} \mathrm{O}_{6}$ ]: C 56.68, H 5.55; Found (\%): C 56.30, H 5.41.

2,5-Dipropylformate hydroquinone (3): Yield: $98.3 \%$. m.p. $100.4{ }^{\circ} \mathrm{C} ;{ }^{1} \mathrm{H}$ NMR $\left(400 \mathrm{~Hz}, \mathrm{CDCl}_{3}\right): \delta 10.11$ (s, $\left.2 \mathrm{H}\right)$, 7.25 (s, 2H), 3.41-3.36 (t, 4H), 1.46-1.30 (m, 4H), 0.94-0.86 $(\mathrm{t}, 6 \mathrm{H})$; IR (KBr, $\left.v_{\max }, \mathrm{cm}^{-1}\right): 3117,2963,2897,1665,1402$, 1208, 1067, 815, 780. Elemental analysis [Anal. calcd. (\%) for $\mathrm{C}_{14} \mathrm{H}_{18} \mathrm{O}_{6}$ ]: C 59.57, H 6.43; Found (\%): C 59.13, H 6.41.

2,5-Diisopropylformate hydroquinone (4): Yield: $97 \%$. m.p. 136.2-137.1 ${ }^{\circ} \mathrm{C} ;{ }^{1} \mathrm{H}$ NMR $\left(400 \mathrm{~Hz}, \mathrm{CDCl}_{3}\right): \delta 12.14(\mathrm{~s}$, 2H), 7.27 (s, 2H), 3.09-2.50 (m, 2H), 1.34-1.24 (d, 12H); IR $\left(\mathrm{KBr}, \mathrm{v}_{\max }, \mathrm{cm}^{-1}\right): 3125,2973,1663,1377,1219,1061,713$, 753. Elemental analysis [Anal. calcd. (\%) for $\mathrm{C}_{14} \mathrm{H}_{18} \mathrm{O}_{6}$ ]: $\mathrm{C}$ 59.57, H 6.43; Found (\%): C 59.04, H 6.13.

2,5-Dibutylformate hydroquinone (5): Yield: $87.9 \%$. m.p. $101.8{ }^{\circ} \mathrm{C} ;{ }^{1} \mathrm{H}$ NMR $\left(400 \mathrm{~Hz}, \mathrm{CDCl}_{3}\right): \delta 12.07$ (s, 2H), $7.21(\mathrm{~s}, 2 \mathrm{H}), 4.184 .15(\mathrm{t}, 4 \mathrm{H}), 1.71-1.58(\mathrm{~m}, 4 \mathrm{H}), 1.45-1.31(\mathrm{~m}$, 4H), 0.93-0.88 (t, 6H); IR (KBr, $\left.v_{\max }, \mathrm{cm}^{-1}\right): 3126,2962,2898$, $1663,1402,1212,1066,814,799$. Elemental analysis [Anal. calcd. (\%) for $\mathrm{C}_{16} \mathrm{H}_{22} \mathrm{O}_{6}$ ]: C 61.92, H 7.15; Found (\%): C 61.84, H 6.89 .

2,5-Di- $\alpha$-methylpropylformate hydroquinone (6): Yield: $85.8 \%$. m.p. $>200{ }^{\circ} \mathrm{C} ;{ }^{1} \mathrm{H}$ NMR $\left(400 \mathrm{~Hz}, \mathrm{CDCl}_{3}\right): \delta$ 12.23 (s, 2H), 7.28 (s, 2H), 2.99-2.74 (m, 2H), 1.94-1.80 (m, $4 \mathrm{H}), 1.46-1.43(\mathrm{~d}, 6 \mathrm{H}), 1.27-1.24(\mathrm{t}, 6 \mathrm{H})$; IR $\left(\mathrm{KBr}, \mathrm{v}_{\max }, \mathrm{cm}^{-1}\right)$ : 3125, 2935, 2565, 1669, 1440, 1217, 1058, 849, 753. Elemental analysis [Anal. calcd. $(\%)$ for $\mathrm{C}_{16} \mathrm{H}_{22} \mathrm{O}_{6}$ ]: $\mathrm{C} 61.92$, H 7.15; Found (\%): C 61.90, H 7.07.

2,5-Diisobutylformate hydroquinone (7): Yield: $86.7 \%$. m.p. $111{ }^{\circ} \mathrm{C} ;{ }^{1} \mathrm{H}$ NMR $\left(400 \mathrm{~Hz}, \mathrm{CDCl}_{3}\right): \delta 12.07$ (s, 2H), 7.29 (s, 2H), 3.97-3.95 (d, 4H), 1.98-1.91 (m, 2H), 0.99-0.88 (d, 12H); IR (KBr, $v_{\max }, \mathrm{cm}^{-1}$ ): 3126, 2968, 2910, 1663, 1407, 1237, 1073, 826, 775. Elemental analysis [Anal. calcd. (\%) for $\mathrm{C}_{16} \mathrm{H}_{22} \mathrm{O}_{6}$ ]: $\mathrm{C}$ 61.92, H 7.15; Found (\%): C 61.57, H 6.94.

2,5-Diamylformate hydroquinone (8): Yield: $83.6 \%$. m.p. $104-105{ }^{\circ} \mathrm{C} ;{ }^{1} \mathrm{H}$ NMR $\left(400 \mathrm{~Hz}, \mathrm{CDCl}_{3}\right): \delta 12.10(\mathrm{~s}, 2 \mathrm{H})$, 7.26 (s, 2H), 4.30-4.27 (t, 4H), 4.18-4.13 (m, 4H), 3.19-2.96 $(\mathrm{m}, 4 \mathrm{H}), 1.73-1.56(\mathrm{~m}, 4 \mathrm{H}), 1.36-1.27(\mathrm{t}, 6 \mathrm{H})$; IR (KBr, $\mathrm{v}_{\max }$, $\mathrm{cm}^{-1}$ ): 3124, 2973, 2896, 1657, 1404, 1226, 1073, 823, 780. Elemental analysis [Anal. calcd. $(\%)$ for $\mathrm{C}_{18} \mathrm{H}_{26} \mathrm{O}_{6}$ ]: $\mathrm{C} 63.89$, H 7.74; Found (\%): C 63.71, H 7.32.

2,5-Diisoamylformate hydroquinone (9): Yield: $80.1 \%$. m.p. $48-49{ }^{\circ} \mathrm{C} ;{ }^{1} \mathrm{H} \mathrm{NMR}\left(400 \mathrm{~Hz}, \mathrm{CDCl}_{3}\right): \delta 12.07$ (s, 2H), $7.26(\mathrm{~s}, 2 \mathrm{H}), 4.34-4.31(\mathrm{t}, 4 \mathrm{H}), 3-2.89(\mathrm{~m}, 4 \mathrm{H}), 1.72-1.60(\mathrm{~m}$, $2 \mathrm{H}), 0.92-0.90$ (d, $12 \mathrm{H})$; IR (KBr, $\left.\mathrm{v}_{\max }, \mathrm{cm}^{-1}\right)$ : 3128, 2959, 2872, 1655, 1401, 1220, 1074, 822, 778. Elemental analysis [Anal. calcd. (\%) for $\mathrm{C}_{18} \mathrm{H}_{26} \mathrm{O}_{6}$ ]: $\mathrm{C} 63.89, \mathrm{H}$ 7.74; Found (\%): C 63.53, H 7.47.

2,5-Dicyclohexylformate hydroquinone (10): Yield: 79.2 \%. m.p. 207-208 ${ }^{\circ} \mathrm{C}$; ${ }^{1} \mathrm{H}$ NMR $\left(400 \mathrm{~Hz}, \mathrm{CDCl}_{3}\right)$ : $\delta 12.24$ (s, 2H), 7.34 (t, 2H), 4-3.98 (m, 2H), 2.03-1.95 (m, 8H), 1.87$1.66(\mathrm{~m}, 8 \mathrm{H}), 1.44-1.27(\mathrm{~m}, 4 \mathrm{H})$; IR $\left(\mathrm{KBr}, \mathrm{v}_{\max }, \mathrm{cm}^{-1}\right)$ : 3048, 2627, 2561, 1646, 1424, 1228, 1058, 836, 749. Elemental analysis [Anal. calcd. (\%) for $\mathrm{C}_{20} \mathrm{H}_{26} \mathrm{O}_{6}$ ]: $\mathrm{C} 66.28, \mathrm{H}$ 7.23; Found (\%): C 66.04, H 7.15.

Antimicrobial activity: The antibacterial activity of the synthesized compounds was tested against Escherichia coli and Bacillus subtilis using MH medium (Mueller-Hinton medium), the antifungal activity of the compounds was tested against Candida albicans using RPMI-1640 mediun. The MICs (minimum inhibitory concentrations) of the test compounds were determined by a colorimetric method using the dye MTT $^{12,13}$. Suspension of the microbes prepared to contain approximately $10^{5} \mathrm{cfu} / \mathrm{mL}$ and applied to 96 -well microplates with serially diluted compounds in DMSO to be tested and incubated at $37{ }^{\circ} \mathrm{C}$ for $24 \mathrm{~h}$ and $48 \mathrm{~h}$ for bacteria and fungi, respectively. After the MICs were visually determined on each of microtitration plates, $50 \mu \mathrm{L}$ MTT solution was added to each well for $4-5 \mathrm{~h}$. The supernatant of each well was removed and $100 \mu \mathrm{L}$ of isopropanol containing $5 \% 1 \mathrm{~mol} / \mathrm{L} \mathrm{HCl}$ was added to extract the dye. The optical density (OD value) was measured with a microplate reader at $550 \mathrm{~nm}$.

\section{RESULTS AND DISCUSSION}

The MICs of the compounds against three bacterias are presented in Table-2. The compounds $\mathbf{2 - 9}$ showed to be inactive against Escherichia coli and Bacillus subtilis. To the contrary, compounds $\mathbf{1}$ and $\mathbf{1 0}$ exhibited antimicrobial activity against the two bacteria strain with an MIC value of $18.9-28.6 \mu \mathrm{g} / \mathrm{mL}$, which was comparable to the positive control kanamycin and penicillin. Although all the inhibiting activities were lower than the positive control, the activities of compounds $\mathbf{1}$ and $\mathbf{1 0}$ exhibited similar antibacterial activities with commercial antibiotics. Compounds 1-10 were also tested against Candida albicans which they had no antifungal activity.

Compounds $\mathbf{1}$ and $\mathbf{1 0}$ showed strong antimicrobial activities. According to structure-activity relationships, it is assumed that the non-substitutional group phenyl ring may play a key role. Other compounds showed no significant inhibition. This may be due to the large substituents hindering the compounds to permeate the cell membrane. Nevertheless, the biological activities of their potential metabolites seems to be worth studying.

\section{Conclusion}

We have synthesized a series of 2,5-substituent hydroquinone derivatives derivatives using a successful method 


\begin{tabular}{|c|c|c|c|}
\hline \multicolumn{4}{|c|}{$\begin{array}{c}\text { TABLE-2 } \\
\text { ANTIMICROBIAL ACTIVITY OF } \\
\text { THE SYNTHESIZED COMPOUNDS }\end{array}$} \\
\hline \multirow[b]{2}{*}{ Compound } & \multicolumn{3}{|c|}{ Minimum inhibitory concentration $[\mu \mathrm{g} / \mathrm{mL}]$} \\
\hline & $\begin{array}{l}\text { Escherichia } \\
\text { coli }\end{array}$ & $\begin{array}{c}\text { Bacillus } \\
\text { subtilis }\end{array}$ & $\begin{array}{l}\text { Candida } \\
\text { albicans }\end{array}$ \\
\hline 1 & $>20.5$ & $>24.6$ & $>50$ \\
\hline 2 & $>50$ & $>50$ & $>50$ \\
\hline 3 & $>50$ & $>50$ & $>50$ \\
\hline 4 & $>50$ & $>50$ & $>50$ \\
\hline 5 & $>50$ & $>50$ & $>50$ \\
\hline 6 & $>50$ & $>50$ & $>50$ \\
\hline 7 & $>50$ & $>50$ & $>50$ \\
\hline 8 & $>50$ & $>50$ & $>50$ \\
\hline 9 & $>50$ & $>50$ & $>50$ \\
\hline 10 & $>18.9$ & $>28.6$ & $>50$ \\
\hline 'Ketoconazole & $>50$ & $>50$ & 3.9 \\
\hline bKanamycin & 3.6 & 3.1 & $>50$ \\
\hline 'Penicillin & $>50$ & 2.6 & $>50$ \\
\hline
\end{tabular}

under mild conditions in a high yield. All the compounds were tested for their antibacterial (Escherichia coli and Bacillus subtilis) and antifungal (Candida albicans) activities by MTT method. It may be concluded that compounds $\mathbf{1}$ and $\mathbf{1 0}$ showed strong antimicrobial activities. The results may be useful to researchers attempting to gain more understanding of the antimicrobial activity of 2,5-substituent hydroquinone compounds.

\section{ACKNOWLEDGEMENTS}

This work was co-financed by grants from the Natural Science Foundation of Weifang (No. 2012098) and National Torch Project, P.R. China (No. 2012GA740034).

\section{REFERENCES}

1. H. Itokawa, N. Totsuka, K. Nakahara, M. Maezuru, K. Takeya, M. Kondo, M. Inamatsu and H. Morita, Chem. Pharm. Bull. (Tokyo), 37, 1619 (1989).

2. J.S. Swenton, S. Patai and Z. Rappoport, The Chemistry of the Quinonoid Compounds; Vol. 2, Wiley, New York (1988).

3. A. Sato, T. Shindo, N. Kasanuki and K. Hasegawa, J. Nat. Prod., 52, 975 (1989).

4. T. Yamamura, K. Nishiwaki, Y. Tanigaki, S. Terauchi, S. Tomiyama and T. Nishiyama, Bull. Chem. Soc. Jpn., 68, 2955 (1995).

5. (a) D.M. Dooley, M.A. McGuirl, D.E. Brown, P.N. Turowski, W.S. Mclntire and P.F. Knowles, Nature, 349, 262 (1991); (b) D.M. Dooley, R.A. Scott, P.F. Knowles, C.M. Colangelo, M.A. McGuirl and D.E. Brown, J. Am. Chem. Soc., 120, 2599 (1998).

6. (a) J.P. Klinman, Chem. Rev., 96, 2541 (1996); (b) W.S. McIntire, Annu. Rev. Nutr., 18, 145 (1998).

7. R. Calvo, E.C. Abresch, R. Bittl, G. Feher, W. Hofbauer, R.A. Isaacson, W. Lubitz, M.Y. Okamura and M. Paddock, J. Am. Chem. Soc., 122, 7327 (2000); C.W. Hoganson, Science, 277, 1953 (1997).

8. (a) D.G. Nichols and S.J. Ferguson, Bioenergetics 2, Academic Press, New York (1992); (b) S. Iwata, Science, 281, 64 (1998).

9. S.X. Wang, M. Mure, K.F. Medzihradsky, A.L. Burlingame, R.A. Brown, D.M. Dooley, A.J. Smith, H.M. Kagan and J.P. Klinman, Science, 273, 1078 (1996).

10. C. Basavaraja, N.R. Kim, H.T. Park and D.S. Huh, Bull. Korean Chem. Soc., 30, 907 (2009).

11. T. Bánsági and O. Steinbock, Chaos, 18, 026102 (2008).

12. P. Cao, X.F. Huang, H. Ding, H.M. Ge, H.Q. Li, B.F. Ruan and H.L. Zhu, Chem. Biodivers., 4, 881 (2007).

13. J. Meletiadis, J.F. Meis, J.W. Mouton, J.P. Donnelly and P.E. Verweij, J. Clin. Microbiol., 38, 2949 (2000). 